\title{
Wavelet based algorithm for numerical study of $(1+2)$-dimensional time fractional diffusion problems
}

\author{
Abdul Ghafoor ${ }^{1}$, Sirajul Haq ${ }^{2}$, Manzoor Hussain ${ }^{3}$ and Poom Kumam ${ }^{4,5^{*}}$
}

\section{"Correspondence:}

poom.kum@kmutt.ac.th

${ }^{4}$ Theoretical and Computational Science (TaCS) Center Department of Mathematics, Faculty of Science, King Mongkuts University of

Technology Thonburi (KMUTT), 126 Pracha Uthit Rd., Bang Mod, Thung

Khru, Bangkok 10140, Thailand

${ }^{5}$ KMUTT-Fixed Point Research

Laboratory, Room SCL 802 Fixed

Point Laboratory, Science

Laboratory Building, Department of

Mathematics, Faculty of Science,

King Mongkut's University of

Technology Thonburi (KMUTT), 126

Pracha-Uthit Road, Bang Mod,

Thrung Khru, Bangkok 10140,

Thailand

Full list of author information is available at the end of the article

\begin{abstract}
An effective and robust scheme is developed for solutions of two-dimensional time fractional heat flow problems. The proposed scheme is based on two-dimensional Haar wavelets coupled with finite differences. The time fractional derivative is approximated by an $L_{1}$-formula while spatial part is approximated by two-dimensional Haar wavelets. The proposed methodology first converts the problem to a discrete form and then with collocation approach to a system of linear equations which is easily solvable. To check the efficiency of the scheme, two error norms, $E_{\infty}$ an $E_{\text {rms, }}$, have been computed. The stability of the scheme has been discussed which is an important part of the manuscript. It is also observed that the spectral radius of the amplification matrix satisfies a stability condition. From computation it is clear that computed results are comparable with the exact solution.
\end{abstract}

Keywords: Diffusion problems; Two-dimensional Haar wavelets; Caputo fractional derivative of constant order; Finite differences

\section{Introduction}

Fractional differential equations (FDEs) play a crucial role in modeling of different physical phenomena such as heat and mass transfer [1, 2], fluid mechanics [3], and financial theory $[4,5]$. Usually these equations arise in the form of fractional partial differential equations (FPDEs). The well known application of FPDEs is their nonlocal property which means that the next state of the dynamical system not only depends on the present state but also on all previous states. That's the reason why fractional calculus has been given more emphasis in the last few decades. One of the most important models of FPDEs is time fractional diffusion equation (TFDE) which is used to describe anomalous diffusion phenomena in transport processes. This model can be obtained from the classical diffusion model by replacing the first order time derivative with a fractional one. Analytical methods have limited ability to solve these problems, therefore, numerical techniques are essential.

In the literature several approaches have been proposed for the solution of TFDEs, for example, finite difference methods [6, 7], spectral method [8], finite element method [9], etc. In contrast to finite difference methods, compact finite difference methods have good

(c) The Author(s) 2020. This article is licensed under a Creative Commons Attribution 4.0 International License, which permits use, sharing, adaptation, distribution and reproduction in any medium or format, as long as you give appropriate credit to the original author(s) and the source, provide a link to the Creative Commons licence, and indicate if changes were made. The images or other third party material in this article are included in the article's Creative Commons licence, unless indicated otherwise in a credit line to the material. If material is not included in the article's Creative Commons licence and your intended use is not permitted by statutory regulation or exceeds the permitted use, you will need to obtain permission directly from the copyright holder. To view a copy of this licence, visit http://creativecommons.org/licenses/by/4.0/. 
accuracy [10, 11], which have been applied for the solution of 1D TFDEs in [12]. For 2D problems, explicit and implicit central difference formulas coupled with extrapolation and kernel based approximation with adaptive technique have been studied in [13, 14]. For multidimensional problems, another method is operator-splitting technique or ADI which transforms a given problem to progressions of $1 \mathrm{D}$ linear problems having low computational cost. For complete details of ADI, the interested readers are referred to the papers $[15,16]$. The idea of the ADI method was extended to FPDEs $[17,18]$.

Among diverse numerical methods, wavelet based methods achieved much attention for solution of FPDEs recently because of ease implementation. Also an interested feature of wavelet based methods is that they are capable of noticing singularities and approximating a function with different resolution levels. There are several families of wavelets, like Daubechies, Symlet, Coiflet, etc. All of them are equally interesting, but Daubechies wavelets of order one, known as Haar wavelets (HW), deserve special attention. HW are composed of piecewise constant functions which attain the values -1 and 1 . These wavelets are good for approximations but have the drawback of discontinuity at the endpoints of an interval. This problem has been discussed by Cattani $[19,20]$ who used interpolating splines to regularize these wavelets. Another approach was used by Chen and Hasio [21], in their work the highest order derivative has been approximated by truncated HW series instead of solution. Further, this approach was used by Lepik [22, 23] to study different problems.

The above discussion about Haar wavelets is limited to integer order differential equations, however, this approach also works for solution of fractional differential equations. Lepik [24] solved fractional integral equations with the help of Haar wavelets. Li [8] applied Haar wavelets for solution of fractional ordinary differential equations (FODEs). Chen et al. [25] analyzed errors of the numerical method for FODEs by Haar wavelets. Ray and Patra [26] solved nonlinear oscillatory van der Pol system using Haar wavelet operational matrices. Yi and Huang [27] implemented an operational matrix method for variable coefficient fractional differential equations. Saeed et al. [28] used Haar wavelet Picard technique for numerical solution of fractional order initial and boundary value problems. Saeed and Rehman [29] applied the same technique for numerical solution of fractional partial differential equations. Majak et al. [30] studied higher order Haar wavelet method for FGM structures. Recently, Alderremya et al. [31] implemented the spectral collocation method together with third order Chebyshev polynomials to certain new models of multispace-fractional Gardner equation. Zhang et al. [32] computed the general solution for impulsive differential equations with Riemann-Liouville fractional-order $q \in(1,2)$. Agarwal and Singh [33] studied mathematical model of Nipah virus with fractional order approach. Morales-Delgado et al. [34] discussed fractional dynamics of oxygen diffusion through capillary to tissues under the influence of external forces considering the fractional operators of Liouville-Caputo and Caputo-Fabrizio. Choi and Agarwal [35] established a fractional integration formula using generalized multiindex Mittag-Leffler function. Agarwal et al. $[36,37]$ studied fixed point results, some new differential equation formulas for extended Mittag-Leffler-type functions and an extension of the Caputo fractional derivative operator involving generalized hypergeometric-type functions. Amiri et al. [38] developed a numerical method based on cosine-trigonometric basis functions to solve Fredholm integral equations of the second kind. Moghadam et al. [39] applied Bernoulli wavelet method for numerical solutions of advection dispersion equations. Farooq et al. [40] used 
Chebyshev wavelet method for solution of delay differential equations. Khalil et al. [41] introduced some new operational matrices of integration for fractional Poisson equations with integral type boundary conditions.

\subsection{Motivation}

Developing methods for solutions of TFDEs either analytical, semianalytical, or numerical is an important task. The motivation of this work is to compute numerical solutions of $2 \mathrm{D}$ TFDEs using two-dimensional Haar wavelets and finite differences. The proposed scheme is hybrid and gives good results which are comparable with exact solutions. Also we will examine the computational stability of the proposed algorithm which is related to the spectral radius of the amplification matrix. The problem which will be consider in this study is defined as follows:

$$
\frac{\partial^{\gamma} C(X, t)}{\partial t^{\gamma}}=\Delta C(X, t)+\mathbf{B}(\mathbf{X}, \mathbf{t}), \quad t>0, X \in \Psi,
$$

together with the initial condition

$$
C(X, 0)=\beta(X), \quad X \in \Psi,
$$

and boundary conditions

$$
C(X, t)=\eta(X, t), \quad t \geq 0, X \in \partial \Psi,
$$

where $X=X(x, y), \Psi$ and $\partial \Psi$ denote solution domain and boundary, $\mathbf{B}(\mathbf{X}, \mathbf{t}), \beta(X), \eta(X, t)$ are known functions, while $C(X, t)$ is an unknown function to be computed. In Eq. (1.1), $\Delta$ is two-dimensional Laplacian and $\frac{\partial^{\gamma} C(X, t)}{\partial t^{\gamma}}$ is the fractional derivative of $C$ with respect to $t$ in Caputo sense which is defined as:

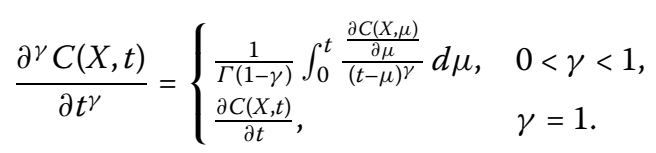

The rest of the paper is organized as follows: Basic definitions of HW and its integrals are reported in Sect. 2. The proposed methodology and convergence with stability analysis have been discussed in Sects. 3 and 4, respectively. Numerical results are given in Sect. 5, and finally conclusion is summarized in Sect. 6.

\section{Preliminaries}

This section is devoted to some basic definitions required in this study. Consider $\mathbb{M}=2^{J}$, where $J$ denotes the maximal resolution level. Next assume $x \in[\mathbb{E}, \mathbb{F}$ ) and partition of the interval into $2 \mathbb{M}$ subintervals of equal length $\Delta x=\frac{\mathbb{F}-\mathbb{E}}{2 \mathbb{M}}$. Further define the wavelet number $i=m+\kappa+1$, where $m=2^{\lambda}, \lambda=0, \ldots, J$ and $\kappa=0, \ldots, 2^{\lambda}-1$, respectively. The Haar wavelets for $i \geq 1$ are defined as follows:

$$
\mathbb{H}_{1}(x)= \begin{cases}1, & x \in[\mathbb{E}, \mathbb{F}) \\ 0, & \text { otherwise }\end{cases}
$$




$$
\mathbb{H}_{i}(x)= \begin{cases}(-1)^{j+1}, & x \in\left[\zeta_{j}(i), \zeta_{j+1}(i)\right), j=1,2 \\ 0, & \text { otherwise }\end{cases}
$$

where $\zeta_{s+1}(i)=\mathbb{E}+(2 \kappa+s) v \delta x, s=0,1,2$ and $v=2^{J-\lambda}$. To solve the $n$th order time fractional partial differential equations (PDEs), one needs repeated integrals of the form

$$
\mathbb{P}_{i, \alpha}(x)=\int_{\mathbb{E}}^{x} \int_{\mathbb{E}}^{x} \ldots \int_{\mathbb{E}}^{x} \mathcal{H}_{i}(y) d y^{\alpha}=\frac{1}{(\alpha-1) !} \int_{\mathbb{E}}^{x}(x-y)^{\alpha-1} \mathbb{H}_{i}(y) d y,
$$

where $\alpha=1,2, \ldots, n, i=1,2, \ldots, 2 \mathbb{M}$. Using Eqs. (2.1), (2.2), and (2.3), the closed-form expression of these integrals are given as [22]:

$$
\begin{aligned}
& \mathbb{P}_{1, \alpha}(x)=\frac{(x-\mathbb{E})^{\alpha}}{\alpha !}, \\
& \mathbb{P}_{i, \alpha}(x)= \begin{cases}0, & x<\zeta_{1}(x), \\
\frac{1}{\alpha !}\left[x-\zeta_{1}(i)\right]^{\alpha}, & x \in\left[\zeta_{1}(i), \zeta_{2}(i)\right), \\
\frac{1}{\alpha !}\left[\left(x-\zeta_{1}(i)\right)^{\alpha}-2\left(\left(x-\zeta_{2}(i)\right)^{\alpha}\right],\right. & x \in\left[\zeta_{2}(i), \zeta_{3}(i)\right), \\
\frac{1}{\alpha !}\left[\left(x-\zeta_{1}(i)\right)^{\alpha}-2\left(\left(x-\zeta_{2}(i)\right)^{\alpha}+\left(x-\zeta_{3}(i)\right)^{\alpha}\right],\right. & x \geq \zeta_{3}(i) .\end{cases}
\end{aligned}
$$

\section{Proposed methodology}

This section is devoted to explaining the proposed methodology for the above mentioned problem. In Eq. (1.4), the time fractional derivative can be approximated by an $L_{1}$-approximation formula [1] as:

$$
\begin{aligned}
\frac{\partial^{\gamma} C\left(X, t^{\xi+1}\right)}{\partial t^{\gamma}} & =\frac{1}{\Gamma(1-\gamma)} \int_{0}^{t^{\xi+1}} \frac{\partial C(X, \mu)}{\partial \mu}\left(t^{\xi+1}-\mu\right)^{-\gamma} d \mu \\
& =\frac{1}{\Gamma(1-\gamma)} \sum_{j=0}^{\xi} \int_{j \tau}^{(j+1) \tau} \frac{\partial C(X, \mu)}{\partial \mu}\left(t^{\xi+1}-\mu\right)^{-\gamma} d \mu \\
& =\frac{1}{\Gamma(1-\gamma)} \sum_{j=0}^{\xi}\left[\frac{C^{j+1}(X)-C^{j}(X)}{\tau}+O(\tau)\right] \int_{j \tau}^{(j+1) \tau}((j+1) \tau-\mu)^{-\gamma} d \mu .
\end{aligned}
$$

A simplification of the above integral yields

$$
\frac{\partial^{\gamma} C}{\partial t^{\gamma}}= \begin{cases}A_{\gamma} \sum_{j=0}^{\xi}\left[C^{\xi-j+1}(X)-C^{\xi-j}(X)\right] \varphi_{\gamma}(j)+O\left(\tau^{2-\gamma}\right), & 0<\gamma<1, \\ \frac{C^{\xi+1}(X)-C^{\xi}(X)}{\tau}+O(\tau), & \gamma=1,\end{cases}
$$

where $A_{\gamma}=\frac{\tau^{-\gamma}}{\Gamma(2-\gamma)}, \varphi_{\gamma}(j)=(j+1)^{1-\gamma}-(j)^{1-\gamma}$. Applying a $\theta$-weighted scheme to Eq. (1.1), we have

$$
\frac{\partial^{\gamma} C}{\partial t^{\gamma}}=\theta[\Delta C(X)]^{\xi+1}+(1-\theta)[\Delta C(X)]^{\xi}+\mathbf{B}^{\xi+1}(X)
$$


Using Eq. (3.1) in Eq. (3.2), one can get

$$
\begin{aligned}
A_{\gamma} C^{\xi+1}(X)-\theta[\Delta C(X)]^{\xi+1}= & A_{\gamma} C^{\xi}(X)-A_{\gamma} \sum_{j=1}^{\xi}\left[C^{\xi-j+1}(X)-C^{\xi-j}(X)\right] \varphi_{\gamma}(j) \\
& +(1-\theta)[\Delta C(X)]^{\xi}+\mathbf{B}^{\xi+1}(X) .
\end{aligned}
$$

In Eq. (3.3), $\Delta=\frac{\partial^{2}}{\partial x^{2}}+\frac{\partial^{2}}{\partial y^{2}}$, therefore we replace mixed derivatives by two-dimensional HW truncated series as

$$
C_{x x y y}^{\xi+1}(x, y)=\sum_{i=1}^{2 \mathbb{M}} \sum_{l=1}^{2 \mathbb{M}} \delta_{i, l}^{\xi+1} \mathbb{H}_{i}(x) \mathbb{H}_{l}(y)
$$

where $\delta_{i, l}^{\xi+1}$ are wavelet coefficients to be calculated. Integration of Eq. (3.4) in the domain $[0, y]$ leads to

$$
C_{x x y}^{\xi+1}(x, y)=\sum_{i=1}^{2 \mathbb{M}} \sum_{l=1}^{2 \mathbb{M}} \delta_{i, l}^{\xi+1} \mathbb{H}_{i}(x) \mathbb{P}_{l, 1}(y)+C_{x x y}^{\xi+1}(x, 0)
$$

Integrating Eq. (3.5) with respect to $y$ from 0 to 1 , the unknown term $C_{x x y}^{\xi+1}(x, 0)$ can be computed as

$$
C_{x x y}^{\xi+1}(x, 0)=C_{x x}^{\xi+1}(x, 1)-C_{x x}^{\xi+1}(x, 0)-\sum_{i=1}^{2 \mathbb{M}} \sum_{l=1}^{2 \mathbb{M}} \delta_{i, l}^{\xi+1} \mathbb{H}_{i}(x) \mathbb{P}_{l, 2}(1)
$$

Substituting Eq. (3.6) into Eq. 3.5), the obtained result is

$$
C_{x x y}^{\xi+1}(x, y)=\sum_{i=1}^{2 \mathbb{M}} \sum_{l=1}^{2 \mathbb{M}} \delta_{i, l}^{\xi+1} \mathbb{H}_{i}(x)\left[\mathbb{P}_{l, 1}(y)-\mathbb{P}_{l, 2}(1)\right]+C_{x x}^{\xi+1}(x, 1)-C_{x x}^{\xi+1}(x, 0) .
$$

Integrating Eq. (3.7) in $[0, y]$, we get

$$
C_{x x}^{\xi+1}(x, y)=\sum_{i=1}^{2 \mathbb{M}} \sum_{l=1}^{2 \mathbb{M}} \delta_{i, l}^{\xi+1} \mathbb{H}_{i}(x)\left[\mathbb{P}_{l, 2}(y)-y \mathbb{P}_{l, 2}(1)\right]+y C_{x x}^{\xi+1}(x, 1)+(1-y) C_{x x}^{\xi+1}(x, 0)
$$

Repeating the same procedure, one can easily derive the following expressions:

$$
\begin{aligned}
C_{y y}^{\xi+1}(x, y)= & \sum_{i=1}^{2 \mathbb{M}} \sum_{l=1}^{2 \mathbb{M}} \delta_{i, l}^{\xi+1}\left[\mathbb{P}_{i, 2}(x)-x \mathbb{P}_{i, 2}(1)\right] \mathbb{H}_{l}(y)+x C_{y y}^{\xi+1}(1, y)+(1-x) C_{y y}^{\xi+1}(0, y), \\
C_{x}^{\xi+1}(x, y)= & \sum_{i=1}^{2 \mathbb{M}} \sum_{l=1}^{2 \mathbb{M}} \delta_{i, l}^{\xi+1}\left[\mathbb{P}_{i, 1}(x)-\mathbb{P}_{i, 2}(1)\right]\left[\mathbb{P}_{l, 2}(y)-y \mathbb{P}_{l, 2}(1)\right]+y C_{x}^{j+1}(x, 1) \\
& +(1-y) C_{x}^{\xi+1}(x, 0)+C^{j+1}(1, y)-C^{j+1}(0, y)-y C^{\xi+1}(1,1)+y C^{\xi+1}(0,1) \\
& +(y-1) C^{\xi+1}(1,0)+(1-y) C^{\xi+1}(0,0),
\end{aligned}
$$




$$
\begin{aligned}
C_{y}^{\xi+1}(x, y)= & \sum_{i=1}^{2 \mathbb{M}} \sum_{l=1}^{2 \mathbb{M}} \delta_{i, l}^{\xi+1}\left[\mathbb{P}_{i, 2}(x)-x \mathbb{P}_{i, 2}(1)\right]\left[\mathbb{P}_{l, 1}(y)-\mathbb{P}_{l, 2}(1)\right]+x C_{y}^{\xi+1}(1, y) \\
& +(1-x) C_{y}^{\xi+1}(0, y)+C^{\xi+1}(x, 1)-C^{\xi+1}(x, 0)-x C^{\xi}(1,1)+x C^{\xi+1}(1,0) \\
& +(x-1) C^{\xi+1}(0,1)+(1-x) C^{\xi+1}(0,0), \\
C^{\xi+1}(x, y)= & \sum_{i=1}^{2 \mathbb{M}} \sum_{l=1}^{2 \mathbb{M}} \delta_{i, l}^{\xi+1}\left[\mathbb{P}_{i, 2}(x)-x \mathbb{P}_{i, 2}(1)\right]\left[\mathbb{P}_{l, 2}(y)-y \mathbb{P}_{l, 2}(1)\right]+y C^{\xi+1}(x, 1) \\
& -y C^{\xi+1}(0,1)+(1-y)\left[C^{\xi+1}(x, 0)-C^{\xi+1}(0,0)\right]+x C^{\xi+1}(1, y) \\
& -x C^{\xi+1}(0, y)-x y\left[C^{\xi+1}(1,1)-C^{\xi+1}(0,1)\right]+x(y-1) C^{\xi+1}(1,0) \\
& +x(1-y) C^{\xi+1}(0,0)+C^{\xi+1}(0, y) .
\end{aligned}
$$

As the proposed method is based on collocation approach, we use the following collocation points:

$$
x_{\omega}=\frac{\omega-0.5}{2 \mathbb{M}}, \quad y_{\varepsilon}=\frac{\varepsilon-0.5}{2 \mathbb{M}}, \quad \text { where } \omega, \varepsilon=1,2, \ldots, 2 \mathbb{M} .
$$

Substituting collocation points and values from Eqs. (3.8), (3.9), and (3.10) into Eq. (3.3) leads to the following system of algebraic equations:

$$
\sum_{i=1}^{2 \mathbb{M}} \sum_{l=1}^{2 \mathbb{M}} \delta_{i, l}^{\xi+1}\left[A_{\gamma} \Omega_{i, l}(\omega, \varepsilon)-\theta \Phi_{i, l}(\omega, \varepsilon)\right]=\Upsilon(\omega, \varepsilon),
$$

where

$$
\begin{aligned}
\Omega_{i, l}(\omega, \varepsilon)= & {\left[\mathbb{P}_{i, 2}\left(x_{\omega}\right)-x_{\omega} \mathbb{P}_{i, 2}(1)\right]\left[\mathbb{P}_{l, 2}\left(y_{\varepsilon}\right)-y_{\varepsilon} \mathbb{P}_{l, 2}(1)\right], } \\
\Phi_{i, l}(\omega, \varepsilon)= & \mathbb{H}_{i}\left(x_{\omega}\right)\left[\mathbb{P}_{l, 2}\left(y_{\varepsilon}\right)-y_{\varepsilon} \mathbb{P}_{l, 2}(1)\right]+\left[\mathbb{P}_{i, 2}\left(x_{\omega}\right)-x_{\omega} \mathbb{P}_{i, 2}(1)\right] \mathbb{H}_{l}\left(y_{\varepsilon}\right), \\
\Upsilon(\omega, \varepsilon)= & A_{\gamma} C^{\xi}\left(x_{\omega}, y_{\varepsilon}\right)-A_{\gamma} \sum_{j=1}^{\xi}\left[C^{\xi-j+1}\left(x_{\omega}, y_{\varepsilon}\right)-C^{\xi-j}\left(x_{\omega}, y_{\varepsilon}\right)\right] \varphi_{\gamma}(j) \\
& +(1-\theta)\left[\Delta C\left(x_{\omega}, y_{\varepsilon}\right)\right]^{\xi}+\mathbf{B}^{\xi+1}\left(x_{\omega}, y_{\varepsilon}\right) \\
& -A_{\gamma}\left[y_{\varepsilon} C^{\xi+1}\left(x_{\omega}, 1\right)-y_{\varepsilon} C^{\xi+1}(0,1)+\left(1-y_{\varepsilon}\right)\left\{C^{\xi+1}\left(x_{\omega}, 0\right)-C^{\xi+1}(0,0)\right\}\right. \\
& +x_{\omega} C^{\xi+1}\left(1, y_{\varepsilon}\right)-x_{\omega} C^{\xi+1}\left(0, y_{\varepsilon}\right)-x_{\omega} y_{\varepsilon}\left\{C^{\xi+1}(1,1)-C^{\xi+1}(0,1)\right\} \\
& \left.+x_{\omega}\left(y_{\varepsilon}-1\right) C^{\xi+1}(1,0)+x_{\omega}\left(1-y_{\varepsilon}\right) C^{\xi+1}(0,0)+C^{\xi+1}\left(0, y_{\varepsilon}\right)\right] \\
& +\theta\left[y_{\varepsilon} C_{x x}^{\xi+1}\left(x_{\omega}, 1\right)+\left(1-y_{\varepsilon}\right) C_{x x}^{\xi+1}\left(x_{\omega}, 0\right)\right. \\
& \left.+x_{\omega} C_{y y}^{\xi+1}\left(1, y_{\varepsilon}\right)+\left(1-x_{\omega}\right) C_{y y}^{\xi+1}\left(0, y_{\varepsilon}\right)\right] .
\end{aligned}
$$

In Eq. (3.11) there are $2 \mathbb{M} \times 2 \mathbb{M}$ equations having matrix size $(2 \mathbb{M})^{2} \times(2 \mathbb{M})^{2}$. After solving the system, an approximate solution can be computed from Eq. (3.10) for arbitrary time.

\section{Convergence and stability}

First we address the convergence theorem of the proposed scheme. The following lemma is necessary for convergence theorem. 
Lemma 1 ([42]) If $f(x, y)$ satisfies a Lipschitz condition on $[0,1] \times[0,1]$, that is, there exists a positive $L$ such that for all $\left(x_{1}, y\right),\left(x_{2}, y\right) \in[0,1] \times[0,1],\left|f\left(x_{1}, y\right)-f\left(x_{2}, y\right)\right| \leq L\left|x_{1}-x_{2}\right|$ then

$$
\delta_{i, l}^{2} \leq \frac{L^{2}}{2^{4 \lambda+4} m^{2}}
$$

Theorem 1 ([43]) Assume $C(x, y)$ and ${ }_{C_{2 M}}(x, y)$ are exact and approximate solution of Eq. (1.1), respectively, then

$$
\left\|E_{J}\right\| \leq \frac{L}{4 \sqrt{255}} \frac{1}{2^{4 j}}
$$

The above result indicates that the resolution level and error norm are inversely proportional. It has been verified that when resolution increases the error norm decreases.

Proof To prove the theorem, take the asymptotic expansion of Eq. (3.10) defined as follows:

$$
C^{\xi+1}(x, y)=\sum_{i=1}^{\infty} \sum_{l=1}^{\infty} \delta_{i, l}^{\xi+1}\left[\mathbb{P}_{i, 2}(x)-x \mathbb{P}_{i, 2}(1)\right]\left[\mathbb{P}_{l, 2}(y)-y \mathbb{P}_{l, 2}(1)\right]+\Lambda(x, y)
$$

where

$$
\begin{aligned}
\Lambda(x, y)= & y C^{\xi+1}(x, 1)-y C^{\xi+1}(0,1)+(1-y)\left[C^{\xi+1}(x, 0)-C^{\xi+1}(0,0)\right] \\
& +x C^{\xi+1}(1, y)-x C^{\xi+1}(0, y)-x y\left[C^{\xi+1}(1,1)-C^{\xi+1}(0,1)\right] \\
& +x(y-1) C^{\xi+1}(1,0)+x(1-y) C^{\xi+1}(0,0)+C^{\xi+1}(0, y) .
\end{aligned}
$$

From Eq. (4.2) at resolution level $J$, we can write

$$
\begin{aligned}
\left|E_{2 \mathbb{M}}\right| & =\left|C(x, y)-C_{2 \mathbb{M}}(x, y)\right| \\
& =\left|\sum_{i=2 \mathbb{M}+1}^{\infty} \sum_{l=2 \mathbb{M}+1}^{\infty} \delta_{i, l}^{\xi+1}\left[\mathbb{P}_{i, 2}(x)-x \mathbb{P}_{i, 2}(1)\right]\left[\mathbb{P}_{l, 2}(y)-y \mathbb{P}_{l, 2}(1)\right]\right| .
\end{aligned}
$$

The $L^{2}$-norm is given by

$$
\begin{aligned}
\left\|E_{2 \mathbb{M}}\right\|^{2} & =\left|\int_{0}^{1} \int_{0}^{1}\left(\sum_{i=2 \mathbb{M}+1}^{\infty} \sum_{l=2 \mathbb{M}+1}^{\infty} \delta_{i, l}^{\xi+1}\left[\mathbb{P}_{i, 2}(x)-x \mathbb{P}_{i, 2}(1)\right]\left[\mathbb{P}_{l, 2}(y)-y \mathbb{P}_{l, 2}(1)\right]\right)^{2} d x d y\right| \\
& =\sum_{i, l=2 \mathbb{M}+1}^{\infty} \sum_{\tilde{i}, \tilde{l}=2 \mathbb{M}+1}^{\infty} \delta_{i, l}^{\xi+1} \delta_{\tilde{i}, \tilde{l}}^{\xi+1}\left|\int_{0}^{1} \int_{0}^{1}\left(\left[\mathbb{P}_{i, 2}(x)-x \mathbb{P}_{i, 2}(1)\right]\left[\mathbb{P}_{l, 2}(y)-y \mathbb{P}_{l, 2}(1)\right]\right)^{2} d x d y\right| \\
& =\sum_{i, l=2 \mathbb{M}+1}^{\infty} \delta_{i, l}^{2}\left|\int_{0}^{1} \int_{0}^{1}\left(\left[\mathbb{P}_{i, 2}(x)-x \mathbb{P}_{i, 2}(1)\right]\left[\mathbb{P}_{l, 2}(y)-y \mathbb{P}_{l, 2}(1)\right]\right)^{2} d x d y\right|
\end{aligned}
$$

To evaluate the complex integral, it is sufficient to estimate the maximum bounds of Haar wavelets integrals which can be obtained from the expression [30]:

$$
\mathbb{P}_{i, n}(x)=\frac{1}{n !}\left[\left(x-\zeta_{1}\right)^{n}-2\left(x-\zeta_{2}\right)^{n}+\left(x-\zeta_{3}\right)^{n}\right]
$$




$$
\begin{aligned}
& =\frac{1}{n !} \sum_{\kappa=2}^{n}\left(\begin{array}{l}
n \\
\kappa
\end{array}\right)\left(x-\zeta_{2}\right)^{n-\kappa}\left[\left(\frac{1}{2^{\lambda+1}}\right)^{\kappa}+\left(\frac{-1}{2^{\lambda+1}}\right)^{\kappa}\right] \\
& \leq \frac{1}{n !} \sum_{\kappa=2}^{n}\left(\begin{array}{l}
n \\
\kappa
\end{array}\right)\left(1-\zeta_{2}\right)^{n-\kappa}\left[\left(\frac{1}{2^{\lambda+1}}\right)^{\kappa}+\left(\frac{-1}{2^{\lambda+1}}\right)^{\kappa}\right] \\
& =\mathbb{P}_{i, n}(1)
\end{aligned}
$$

From Eq. (4.5), one can deduce that

$$
\mathbb{P}_{i, 2}(1) \leq \frac{1}{\left(2^{\lambda+1}\right)^{2}} .
$$

Using Eq. (4.7) and the above lemma in Eq. (4.4), we can derive

$$
\begin{aligned}
\left\|E_{2 \mathbb{M}}\right\|^{2} & \leq \sum_{i, l=2 \mathbb{M}+1}^{\infty} \frac{16 L^{2}}{2^{4 \lambda+4} 2^{2 \lambda}} \int_{\zeta_{1}}^{1} \int_{\zeta_{1}}^{1} \frac{1}{2^{4 \lambda+4}} d x d y \\
& =\sum_{i, l=2 \mathbb{M}+1}^{\infty} \frac{16 L^{2}}{2^{10 \lambda+8}} \int_{\zeta_{1}}^{1}\left(1-\zeta_{1}\right) d y \\
& \leq \sum_{i, l=2 \mathbb{M}+1}^{\infty} \frac{16 L^{2}}{2^{10 \lambda+8}} \int_{\zeta_{1}}^{1} d y \\
& \leq \sum_{i, l=2 \mathbb{M}+1}^{\infty} \frac{16 L^{2}}{2^{10 \lambda+8}} \\
& =\sum_{\lambda=J+1}^{\infty}\left\{\sum_{i=0}^{2^{\lambda}-1} \sum_{l=0}^{2^{\lambda}-1} \frac{16 L^{2}}{2^{10 \lambda+8}}\right\} \\
& =\sum_{\lambda=J+1}^{\infty} \frac{16 L^{2}}{2^{8 \lambda+8}},
\end{aligned}
$$

which gives

$$
\left\|E_{2 \mathbb{M}}\right\| \leq \frac{L}{4 \sqrt{255}} \frac{1}{2^{4 J}} .
$$

This completes the proof of the theorem.

\subsection{Stability}

Here stability analysis of the proposed scheme is given. In matrix form, Eqs. (3.8), (3.9) and (3.10) can be written as

$$
\begin{aligned}
& C_{x x}^{\xi+1}=\mathcal{D} \delta^{\xi+1}+\tilde{\mathcal{D}}^{\xi+1}, \\
& C_{y y}^{\xi+1}=\mathcal{E} \delta^{\xi+1}+\tilde{\mathcal{E}}^{\xi+1} \\
& C^{\xi+1}=\mathcal{F} \delta^{\xi+1}+\tilde{\mathcal{F}}^{\xi+1},
\end{aligned}
$$

where $\delta^{\xi+1}=\delta^{\xi+1}(i, l), \mathcal{D}, \mathcal{E}, \mathcal{F}$ and $\tilde{\mathcal{D}}^{\xi+1}, \tilde{\mathcal{E}}^{\xi+1}, \tilde{\mathcal{F}}^{\xi+1}$ are interpolation matrices of $C_{x x}^{\xi+1}$, $C_{y y}^{\xi+1}, C^{\xi+1}$ at collocation points and boundary terms, respectively. Now using Eqs. (4.11), 
(4.12), and (4.13) in Eq. (3.3), we get

$$
\left[A_{\gamma} \mathcal{F}-\theta(\mathcal{D}+\mathcal{E})\right] \delta^{\xi+1}=\left[A_{\gamma} \mathcal{F}+(1-\theta)(\mathcal{D}+\mathcal{E})\right] \delta^{\xi}+\mathcal{G}^{\xi+1}
$$

where

$$
\begin{aligned}
\mathcal{G}^{\xi+1}= & -A_{\gamma} \tilde{\mathcal{F}}^{\xi+1}+\theta\left(\tilde{\mathcal{D}}^{\xi+1}+\tilde{\mathcal{E}}^{\xi+1}\right)+A_{\gamma} \tilde{\mathcal{F}}^{\xi}+(1-\theta)\left(\tilde{\mathcal{D}}^{\xi}+\tilde{\mathcal{E}}^{\xi}\right) \\
& +\mathbf{B}^{\xi+1}-A_{\gamma} \sum_{j=1}^{\xi}\left[C^{\xi-j+1}(X)-C^{\xi-j}(X)\right] \varphi_{\gamma}(j) .
\end{aligned}
$$

From Eq. (4.14) one can write

$$
\delta^{\xi+1}=\mathcal{M}^{-1} \mathcal{N} \delta^{\xi}+\mathcal{M}^{-1} \mathcal{G}^{\xi+1}
$$

where $\mathcal{M}=\left[A_{\gamma} \mathcal{F}-\theta(\mathcal{D}+\mathcal{E})\right], \mathcal{N}=\left[A_{\gamma} \mathcal{F}+(1-\theta)(\mathcal{D}+\mathcal{E})\right]$. Putting Eq. (4.15) in Eq. (4.13), we get

$$
C^{\xi+1}=\mathcal{F} \mathcal{M}^{-1} \mathcal{N} \delta^{\xi}+\mathcal{F} \mathcal{M}^{-1} \mathcal{G}^{\xi+1}+\tilde{\mathcal{F}}^{\xi+1}
$$

Using Eq. (4.13) in Eq. (4.16), we have

$$
C^{\xi+1}=\mathcal{F} \mathcal{M}^{-1} \mathcal{N} \mathcal{F}^{-1} C^{\xi}-\mathcal{F} \mathcal{M}^{-1} \mathcal{N} \mathcal{F}^{-1} \tilde{\mathcal{F}}^{\xi}+\mathcal{F} \mathcal{M}^{-1} \mathcal{G}^{\xi+1}+\tilde{\mathcal{F}}^{\xi+1}
$$

The above equation shows a recurrence relation of the full discretization scheme which allows us refinement in time. If $\tilde{C}^{\xi+1}$ is a numerical solution then

$$
\tilde{C}^{\xi+1}=\mathcal{F} \mathcal{M}^{-1} \mathcal{N} \mathcal{F}^{-1} \tilde{C} \xi-\mathcal{F} \mathcal{M}^{-1} \mathcal{N} \mathcal{F}^{-1} \tilde{\mathcal{F}}^{\xi}+\mathcal{F} \mathcal{M}^{-1} \mathcal{G}^{\xi+1}+\tilde{\mathcal{F}}^{\xi+1}
$$

Let $e^{\xi}=C^{\xi}-\tilde{C}^{\xi}$ be the error at the $\xi$ th time level. Subtracting Eq. (4.17) from Eq. (4.18) then gives

$$
e^{\xi+1}=\mathcal{T} e^{\xi}
$$

where $\mathcal{T}=\mathcal{F} \mathcal{M}^{-1} \mathcal{N F}^{-1}$ is the amplification matrix. According to Lax-Richtmyer criterion, the scheme will be stable if $\|\mathcal{T}\| \leq 1$.

\section{Illustrative test problems}

In this section of the manuscript, we solve some test problems to validate the proposed method. To check goodness, we compute the error norms, $E_{\infty}$ and $E_{\mathrm{rms}}$, which are defined as follows:

$$
E_{\infty}=\max _{1 \leq i, j, \leq 2 \mathbb{M}}\left\|C_{i, j}^{\mathrm{ext}}-C_{i, j}^{\mathrm{app}}\right\|, \quad E_{\mathrm{rms}}=\sqrt{\frac{1}{2 \mathbb{M} \times 2 \mathbb{M}} \sum_{i=1}^{2 \mathbb{M}} \sum_{j=1}^{2 \mathbb{M}}\left(C_{i, j}^{\mathrm{ext}}-C_{i, j}^{\mathrm{app}}\right)^{2}},
$$


Table 1 Error norms of Example 5.1 at $J=4, \tau=0.01, \theta=1$

\begin{tabular}{|c|c|c|c|c|c|}
\hline \multirow[t]{2}{*}{$\gamma$} & \multicolumn{2}{|l|}{$t=0.1$} & \multicolumn{2}{|l|}{$t=0.2$} & \multirow[t]{2}{*}{ Spectral radius } \\
\hline & $\overline{E_{\infty}}$ & $E_{\mathrm{rms}}$ & $E_{\infty}$ & $E_{\mathrm{rms}}$ & \\
\hline 0.1 & $1.8877 \mathrm{e}-04$ & $9.6606 \mathrm{e}-05$ & $9.7649 \mathrm{e}-04$ & $4.9977 \mathrm{e}-04$ & 0.0036 \\
\hline 0.3 & $1.3275 e-04$ & $6.7857 \mathrm{e}-05$ & $9.6571 \mathrm{e}-04$ & $4.9420 \mathrm{e}-04$ & 0.0075 \\
\hline 0.5 & $6.4562 \mathrm{e}-05$ & $3.3075 \mathrm{e}-05$ & $6.4964 \mathrm{e}-04$ & $3.3163 \mathrm{e}-04$ & 0.0117 \\
\hline 0.7 & $5.3845 \mathrm{e}-04$ & $2.7448 \mathrm{e}-04$ & $3.5560 \mathrm{e}-04$ & $1.8222 \mathrm{e}-04$ & 0.0122 \\
\hline 0.9 & $1.3669 \mathrm{e}-03$ & $6.8883 e-04$ & $2.5675 \mathrm{e}-03$ & $1.3077 \mathrm{e}-03$ & 0.0089 \\
\hline
\end{tabular}

Table 2 Error norms of Example 5.1 at $\gamma=0.75, \theta=1$

\begin{tabular}{llll}
\hline$\tau$ & $t=0.2$ & & Spectral radius \\
\cline { 2 - 3 } & $E_{\infty}$ & $E_{\text {rms }}$ & \\
\hline$\frac{1}{10}$ & $1.5323 \mathrm{e}-03$ & $7.7882 \mathrm{e}-04$ & 0.0092 \\
$\frac{1}{20}$ & $1.5083 \mathrm{e}-03$ & $7.6890 \mathrm{e}-04$ & 0.0114 \\
$\frac{1}{40}$ & $1.2385 \mathrm{e}-03$ & $6.3335 \mathrm{e}-04$ & 0.0126 \\
$\frac{1}{80}$ & $8.9192 \mathrm{e}-04$ & $4.5632 \mathrm{e}-04$ & 0.0121 \\
$\frac{1}{160}$ & $5.3891 \mathrm{e}-04$ & $2.7594 \mathrm{e}-04$ & 0.0103 \\
\hline
\end{tabular}

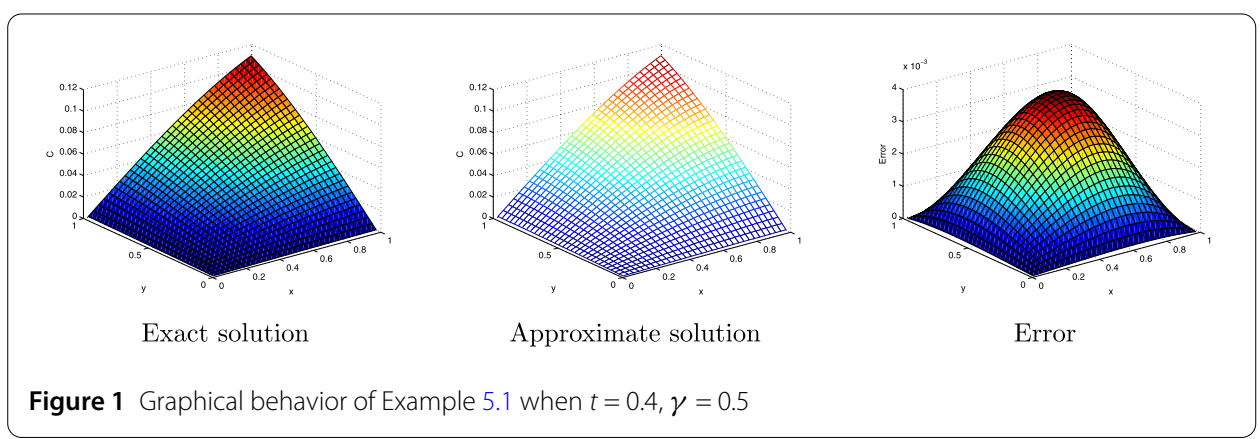

Example 5.1 Consider Eq. (1.1) with source term $\mathbf{B}(x, y, t)=\left[\frac{2 t^{2-t}}{\Gamma(3-\gamma)}+2 t^{2}\right] \sin (x) \sin (y)$. Initial and boundary conditions (Eqs. (1.2)-(1.3)) have been extracted from the exact solution $C(x, y, t)=t^{2} \sin (x) \sin (y)$. The problem has been solved for $\tau=0.01, J=4, \gamma=$ $0.1,0.3,0.5,0.7,0.9$ and the obtained results tabulated in Table 1 . In Table 2 calculated error norms with decreasing time step size have been given, which show that the scheme is convergent in time. In Tables 1 and 2, the spectral radius of the amplification matrix has been mentioned. It is obvious from the tables that computed solutions agree with exact, and the spectral radius lies in the stability domain. Solution profile and absolute errors are plotted in Fig. 1. From the figure it is clear that the proposed solutions agree well with the exact solution.

Example 5.2 Consider Eq. (1.1) with the exact solution $C(x, y, t)=\exp (x+y) t^{1+\gamma}$. Initial and boundary conditions have been used from the exact solution. The source term $\mathbf{B}(x, y, t)$ is easy to derive corresponding to the exact solution. The problem has been solved for different times, and the obtained results have been reported in Table 3. In this problem, we fix $t=0.2, \gamma=0.9$, while varying resolution level $J$. The achieved results are presented in Table 4. One can see clearly that when the resolution level increases the accuracy also increases which guarantees spatial convergence. The same table reveals that the spectral radius remains the same when the resolution level varies. To clarify the spatial convergence 
Table 3 Error norms of Example 5.2 at $J=4, \tau=0.001, \theta=1$

\begin{tabular}{|c|c|c|c|c|c|}
\hline \multirow[t]{2}{*}{$\gamma$} & \multicolumn{2}{|l|}{$t=0.01$} & \multicolumn{2}{|l|}{$t=0.1$} & \multirow[t]{2}{*}{ Spectral radius } \\
\hline & $E_{\infty}$ & $E_{\mathrm{rms}}$ & $E_{\infty}$ & $E_{\mathrm{rms}}$ & \\
\hline 0.1 & $1.7327 \mathrm{e}-03$ & $9.5471 \mathrm{e}-04$ & $2.2000 \mathrm{e}-02$ & $1.2113 \mathrm{e}-02$ & 0.0043 \\
\hline 0.3 & $1.6562 \mathrm{e}-03$ & $9.1517 \mathrm{e}-04$ & $2.3715 \mathrm{e}-02$ & $1.3060 \mathrm{e}-02$ & 0.0107 \\
\hline 0.5 & $1.2383 \mathrm{e}-03$ & $6.9353 \mathrm{e}-04$ & $2.4459 \mathrm{e}-02$ & $1.3481 \mathrm{e}-02$ & 0.0116 \\
\hline 0.7 & $5.2069 e-04$ & $2.9839 \mathrm{e}-04$ & $2.1145 \mathrm{e}-02$ & $1.1700 \mathrm{e}-02$ & 0.0054 \\
\hline 0.9 & $1.1861 \mathrm{e}-04$ & $6.6477 \mathrm{e}-05$ & $8.6087 \mathrm{e}-03$ & $4.8368 \mathrm{e}-03$ & 0.0017 \\
\hline
\end{tabular}

Table 4 Error norms of Example 5.2 at $\gamma=0.9, \theta=1$

\begin{tabular}{llll}
\hline$J$ & $t=0.2$ & & Spectral radius \\
\cline { 2 - 3 } & $E_{\infty}$ & $E_{\text {rms }}$ & \\
\hline 1 & $1.4207 \mathrm{e}-02$ & $8.0262 \mathrm{e}-03$ & 0.0017 \\
2 & $1.4021 \mathrm{e}-02$ & $7.7910 \mathrm{e}-03$ & 0.0017 \\
3 & $1.3964 \mathrm{e}-02$ & $7.7374 \mathrm{e}-03$ & 0.0017 \\
4 & $1.3949 \mathrm{e}-02$ & $7.7244 \mathrm{e}-03$ & 0.0017 \\
\hline
\end{tabular}

Table 5 Absolute errors at different points of Example 5.2 at $t=0.2, \gamma=0.9$

\begin{tabular}{|c|c|c|c|c|}
\hline \multirow[t]{2}{*}{$(x, y)$} & $J=1$ & $J=2$ & $J=3$ & $J=4$ \\
\hline & $E_{\mathrm{abs}}$ & $E_{\mathrm{abs}}$ & $E_{\mathrm{abs}}$ & $E_{\mathrm{abs}}$ \\
\hline$(0.2,0.2)$ & $4.39 e-03$ & $4.25 e-03$ & $4.22 \mathrm{e}-03$ & $4.21 \mathrm{e}-03$ \\
\hline$(0.3,0.3)$ & $7.56 \mathrm{e}-03$ & $7.38 \mathrm{e}-03$ & $7.33 e-03$ & $7.32 \mathrm{e}-03$ \\
\hline$(0.4,0.4)$ & $1.04 \mathrm{e}-02$ & $1.02 \mathrm{e}-02$ & $1.01 \mathrm{e}-02$ & $1.01 \mathrm{e}-02$ \\
\hline$(0.5,0.5)$ & $1.25 \mathrm{e}-02$ & $1.22 \mathrm{e}-02$ & $1.21 \mathrm{e}-02$ & $1.21 \mathrm{e}-02$ \\
\hline$(0.6,0.6)$ & $1.32 \mathrm{e}-02$ & $1.28 \mathrm{e}-02$ & $1.28 \mathrm{e}-02$ & $1.27 \mathrm{e}-02$ \\
\hline$(0.7,0.7)$ & $1.21 \mathrm{e}-02$ & $1.18 \mathrm{e}-02$ & $1.17 \mathrm{e}-02$ & $1.17 \mathrm{e}-02$ \\
\hline$(0.8,0.8)$ & $9.47 \mathrm{e}-03$ & $8.90 e-03$ & $8.80 \mathrm{e}-03$ & $8.78 \mathrm{e}-03$ \\
\hline
\end{tabular}

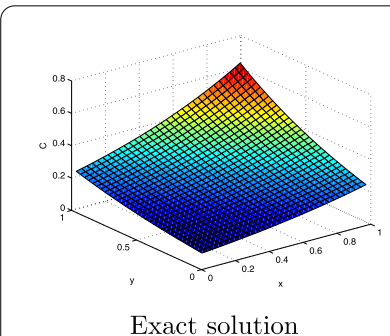

Exact solution

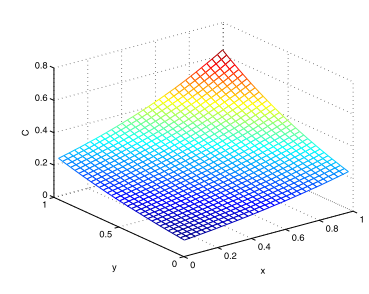

Approximate solution

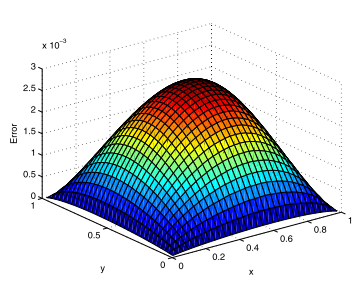

Error

Figure 2 Graphical behavior of Example 5.2 when $t=0.2, \gamma=0.5$

more, the absolute errors $E_{\mathrm{abs}}$ at different mesh points have been addressed in Table 5 . Graphical solutions together with absolute errors are shown in Fig. 2. From the figure it is obvious that the obtained results and exact solutions have strong agreement.

Example 5.3 Consider Eq. (1.1) with the exact solution $C(x, y, t)=t^{2}\left(x-x^{2}\right)^{2}\left(y-y^{2}\right)^{2}$. The source term is easy to compute from the exact solution. In Table 6, we recorded the computed error norms for parameters $t=0.5,0.1, \gamma=0.2,0.4,0.6,0.8, \tau=0.01$. In Fig. 3, exact versus approximate solutions and absolute errors have been plotted. From tabulated data and graphical solution, it is clear that computed solutions match well with the exact one. 
Table 6 Error norms of Example 5.3 at $J=4, \tau=0.01, \theta=1$

\begin{tabular}{|c|c|c|c|c|c|}
\hline \multirow[t]{2}{*}{$\gamma$} & \multicolumn{2}{|l|}{$t=0.5$} & \multicolumn{2}{|l|}{$t=1$} & \multirow[t]{2}{*}{ Spectral radius } \\
\hline & $E_{\infty}$ & $E_{\mathrm{rms}}$ & $E_{\infty}$ & $E_{\mathrm{rms}}$ & \\
\hline 0.2 & $9.5445 \mathrm{e}-05$ & $4.5257 \mathrm{e}-05$ & $3.3886 \mathrm{e}-04$ & $1.6066 \mathrm{e}-04$ & 0.0053 \\
\hline 0.4 & $1.0763 e-04$ & $5.1031 \mathrm{e}-05$ & $3.3842 \mathrm{e}-04$ & $1.6043 \mathrm{e}-04$ & 0.0098 \\
\hline 0.6 & $1.0743 e-04$ & $5.0906 \mathrm{e}-05$ & $3.0342 \mathrm{e}-04$ & $1.4377 \mathrm{e}-04$ & 0.0126 \\
\hline 0.8 & 7.9328e-05 & $3.7517 \mathrm{e}-05$ & $2.0499 \mathrm{e}-04$ & $9.6982 \mathrm{e}-05$ & 0.0108 \\
\hline
\end{tabular}

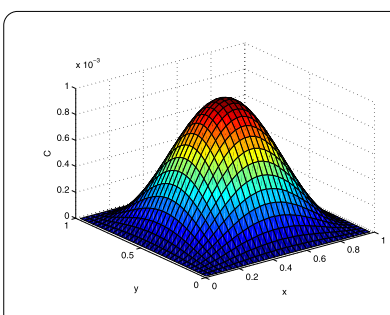

Exact solution

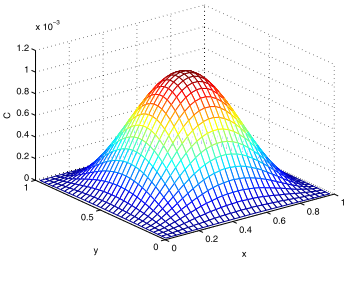

Approximate solution

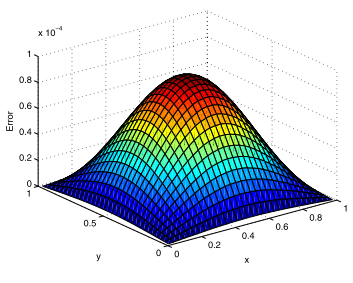

Error

Figure 3 Graphical behavior of Example 5.3 when $t=0.5, \gamma=0.75$

Table 7 Error norms of Example 5.4 at $J=3, \theta=1$

\begin{tabular}{llllll}
\hline$\gamma$ & $t=0.1$ & & & $t=1$ & \\
\cline { 2 - 3 } & $E_{\infty}$ & $E_{\text {rms }}$ & & & $E_{\infty}$ \\
\hline 0.1 & $8.8318 \mathrm{e}-03$ & $6.4683 \mathrm{e}-04$ & & $9.7883 \mathrm{e}-02$ & $7.2690 \mathrm{e}-04$ \\
0.3 & $8.1832 \mathrm{e}-03$ & $5.8760 \mathrm{e}-04$ & & $9.8313 \mathrm{e}-02$ & $7.3059 \mathrm{e}-04$ \\
0.5 & $7.2627 \mathrm{e}-03$ & $5.1105 \mathrm{e}-04$ & & $9.8753 \mathrm{e}-02$ & $7.3507 \mathrm{e}-04$ \\
0.7 & $6.7611 \mathrm{e}-03$ & $4.7713 \mathrm{e}-04$ & & $9.9246 \mathrm{e}-02$ & $7.4066 \mathrm{e}-04$ \\
\hline
\end{tabular}

Example 5.4 Now we consider a nonlinear time fractional differential equation of the form

$$
\frac{\partial^{\gamma} C(X, t)}{\partial t^{\gamma}}=\Delta C(X, t)+2 C(X, t)-C^{2}(X, t)+\mathbf{B}(\mathbf{X}, \mathbf{t}), \quad t>0, X \in \Psi,
$$

where

$$
\begin{aligned}
\mathbf{B}(\mathbf{X}, \mathbf{t})= & \frac{\sin (x) \sin (\gamma)}{4}\left[\frac{\Gamma(2.4)}{\Gamma(2.4-\gamma)} t^{(1.4-\gamma)}+\frac{\Gamma(2.2)}{\Gamma(2.2-\gamma)} t^{(1.2-\gamma)}+\frac{\Gamma(2)}{\Gamma(2-\gamma)} t^{1-\gamma}\right] \\
& +\sin ^{2}(x) \sin ^{2}(y)\left(t^{1.4}+t^{1.2}+t+1\right)^{2} .
\end{aligned}
$$

The associated initial and boundary conditions have been extracted from the exact solution

$$
C(x, y, t)=\frac{\sin (x) \sin (y)\left(t^{1.4}+t^{1.2}+t+1\right)}{4} .
$$

In Table 7, we presented computed error norms for the nonlinear problem at different values of $\gamma$. From the table it is obvious that the current scheme also works for twodimensional nonlinear time fractional problems. Graphical solutions together with absolute errors have been shown Fig. 4. From the figure one can observe that computed solutions match well with the exact solution. 


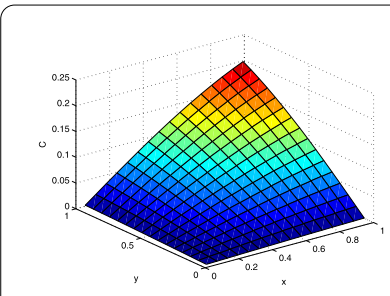

Exact solution

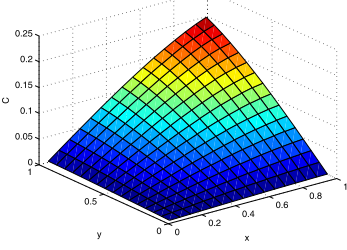

Approximate solution

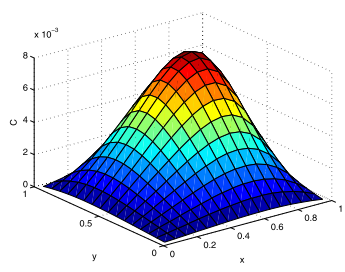

Error

Figure 4 Graphical behavior of Example 5.4 when $t=0.1, \gamma=0.9$

Table 8 Error norms of Example 5.5 at $J=3$

\begin{tabular}{lll}
\hline$\tau$ & $t=1$ & \\
\cline { 2 - 3 } & $E_{\infty}$ & $E_{\text {rms }}$ \\
\hline $1 / 10$ & $7.0810 \mathrm{e}-02$ & $4.6813 \mathrm{e}-02$ \\
$1 / 20$ & $6.8423 \mathrm{e}-02$ & $2.3741 \mathrm{e}-02$ \\
$1 / 40$ & $6.5249 \mathrm{e}-02$ & $1.1568 \mathrm{e}-02$ \\
$1 / 80$ & $6.1135 \mathrm{e}-02$ & $5.4704 \mathrm{e}-03$ \\
$1 / 160$ & $5.6146 \mathrm{e}-02$ & $2.5193 \mathrm{e}-03$ \\
\hline
\end{tabular}

Example 5.5 Finally, we consider a nonlinear time fractional differential equation of the form

$$
\frac{\partial^{\gamma} C(X, t)}{\partial t^{\gamma}}=\Delta C(X, t)+C(X, t)-C^{2}(X, t)+\mathbf{B}(\mathbf{X}, \mathbf{t}), \quad t>0, X \in \Psi,
$$

where

$$
\begin{aligned}
\mathbf{B}(\mathbf{X}, \mathbf{t})= & \left(\Gamma(1+\gamma)+\frac{\Gamma(3)}{\Gamma(3-\gamma)} t^{(2-\gamma)}\right) x^{2}(1-x)^{2} y^{2}(1-y)^{2} \\
& -2\left[(1-x)^{2}-4 x(1-x)+x^{2}+(1-y)^{2}-4 y(1-y)+y^{2}\right] \\
& -\left(t^{\gamma}+t^{2}\right)\left(x^{2}(1-x)^{2} y^{2}(1-y)^{2}\right)\left(1-\left(t^{\gamma}+t^{2}\right)\left(x^{2}(1-x)^{2} y^{2}(1-y)^{2}\right)\right) .
\end{aligned}
$$

The exact solution of this problem is $C(x, y, t)=\left(t^{\gamma}+t^{2}\right) x^{2}(1-x)^{2} y^{2}(1-y)^{2}$. It is obvious that the first derivative of the exact solution approaches infinity when $t \rightarrow 0$, which shows that there is an initial singularity in time. Computed results have been addressed in Table 8 for $J=3$. From the table it is clear that the suggested scheme works if an initial singularity exists in time. Also the same table shows time convergence of the suggested scheme for a singularity problem.

\section{Concluding remarks}

In this work, we proposed a numerical scheme based on two-dimensional Haar wavelets combined with finite differences for solution of time fractional diffusion equations. The scheme has been applied for solutions of five test problems and noted the achieved results in tabulated as well as in graphical form. It has been observed that two-dimensional Haar wavelets work well for solution of two-dimensional time fractional heat problems. 


\section{Funding}

The research work is supported by the Center of Excellence in Theoretical and Computational Science (TaCS-CoE), Faculty of Science, King Mongkut's University of Technology Thonburi (KMUTT).

\section{Availability of data and materials}

Not applicable.

\section{Competing interests}

The authors declare that they have no conflict of interest.

\section{Authors' contributions}

The idea of this paper was proposed by AG and prepared the manuscript initially. All authors equally contributed in this article. All authors read and approved the final manuscript.

\section{Author details}

${ }^{1}$ Institute of Numerical Sciences, Kohat University of Science and Technology, Kohat, 26000, KP, Pakistan. ${ }^{2}$ Faculty of Engineering Sciences, GIK Institute, Topi, 23640, KP, Pakistan. ${ }^{3}$ Department of Humanities and Science, College of Aeronautical Engineering, National University of Science and Tecnology, PAF Academy Asghar Khan, Risalpur, Pakistan. ${ }^{4}$ Theoretical and Computational Science (TaCS) Center Department of Mathematics, Faculty of Science, King Mongkuts University of Technology Thonburi (KMUTT), 126 Pracha Uthit Rd., Bang Mod, Thung Khru, Bangkok 10140, Thailand. ${ }^{5}$ KMUTT-Fixed Point Research Laboratory, Room SCL 802 Fixed Point Laboratory, Science Laboratory Building, Department of Mathematics, Faculty of Science, King Mongkut's University of Technology Thonburi (KMUTT), 126 Pracha-Uthit Road, Bang Mod, Thrung Khru, Bangkok 10140, Thailand.

\section{Publisher's Note}

Springer Nature remains neutral with regard to jurisdictional claims in published maps and institutional affiliations.

Received: 25 January 2020 Accepted: 27 July 2020 Published online: 18 August 2020

\section{References}

1. Hussain, M., Haq, S.: Weighted meshless spectral method for the solutions of multi-term time fractional advection-diffusion problems arising in heat and mass transfer. Int. J. Heat Mass Transf. 129, 1305-1316 (2019)

2. Qi, H.-T., Xu, H.-Y., Guo, X.-W.: The Cattaneo-type time fractional heat conduction equation for laser heating. Comput. Math. Appl. 66(5), 824-831 (2013)

3. Podlubny, I.: Fractional Differential Equations: An Introduction to Fractional Derivatives, Fractional Differential Equations, to Methods of Their Solution and Some of Their Applications. Elsevier, Amsterdam (1998)

4. Raberto, M., Scalas, E., Mainardi, F.: Waiting-times and returns in high-frequency financial data: an empirical study. Phys. A, Stat. Mech. Appl. 314(1-4), 749-755 (2002)

5. Sabatelli, L., Keating, S., Dudley, J., Richmond, P.: Waiting time distributions in financial markets. Eur. Phys. J. B, Condens. Matter Complex Syst. 27(2), 273-275 (2002)

6. Sun, Z., Li, X.: A compact alternating direction implicit difference method for reaction diffusion equations. Math. Numer. Sin. 27(2), 209-224 (2005)

7. Tadjeran, C., Meerschaert, M.M.: A second-order accurate numerical method for the two-dimensional fractional diffusion equation. J. Comput. Phys. 220(2), 813-823 (2007)

8. Li, X., Xu, C.: A space-time spectral method for the time fractional diffusion equation. SIAM J. Numer. Anal. 47(3), 2108-2131 (2009)

9. Ervin, V.J., Heuer, N., Roop, J.P.: Numerical approximation of a time dependent, nonlinear, space-fractional diffusion equation. SIAM J. Numer. Anal. 45(2), 572-591 (2007)

10. Hirsh, R.S.: Higher order accurate difference solutions of fluid mechanics problems by a compact differencing technique. J. Comput. Phys. 19(1), 90-109 (1975)

11. Lele, S.K.: Compact finite difference schemes with spectral-like resolution. J. Comput. Phys. 103(1), 16-42 (1992)

12. Cui, M.: Compact finite difference method for the fractional diffusion equation. J. Comput. Phys. 228(20), 7792-7804 (2009)

13. Brunner, H., Ling, L., Yamamoto, M.: Numerical simulations of $2 d$ fractional subdiffusion problems. J. Comput. Phys. 229(18), 6613-6622 (2010)

14. Chen, C.-M., Liu, F., Turner, I., Anh, V.: Numerical schemes and multivariate extrapolation of a two-dimensional anomalous sub-diffusion equation. Numer. Algorithms 54(1), 1-21 (2010)

15. Douglas, J.: A numerical method for the solution of a parabolic system. Mathematical Sciences Directorate, Office of Scientific Research, US Air Force (1960)

16. Dendy, J. Jr: An alternating direction method for Schrodinger's equation. SIAM J. Numer. Anal. 14(6), 1028-1032 (1977)

17. Wang, H., Wang, K.: An o $\left(n \log _{2} n\right)$ alternating-direction finite difference method for two-dimensional fractional diffusion equations. J. Comput. Phys. 230(21), 7830-7839 (2011)

18. Zhang, Y.-N., Sun, Z.-Z:: Alternating direction implicit schemes for the two-dimensional fractional sub-diffusion equation. J. Comput. Phys. 230(24), 8713-8728 (2011)

19. Cattani, C.: Haar wavelet splines. J. Interdiscip. Math. 4(1), 35-47 (2001)

20. Cattani, C.: Haar wavelets based technique in evolution problems. In: Proceedings Estonian Academy of Sciences Physics Mathematics, vol. 53, pp. 45-63. Estonian Academy Publishers; 1999 (2004)

21. Chen, C., Hsiao, C.: Haar wavelet method for solving lumped and distributed-parameter systems. IEE Proc., Contro Theory Appl. 144(1), 87-94 (1997) 
22. Lepik, Ü.: Numerical solution of evolution equations by the Haar wavelet method. Appl. Math. Comput. 185(1), 695-704 (2007)

23. Lepik, Ü: Solving PDEs with the aid of two-dimensional Haar wavelets. Comput. Math. Appl. 61(7), 1873-1879 (2011)

24. Lepik, Ü.: Solving fractional integral equations by the Haar wavelet method. Appl. Math. Comput. 214(2), 468-478 (2009)

25. Chen, Y., Yi, M., Yu, C.: Error analysis for numerical solution of fractional differential equation by Haar wavelets method. J. Comput. Sci. 3(5), 367-373 (2012)

26. Ray, S.S., Patra, A.: Haar wavelet operational methods for the numerical solutions of fractional order nonlinear oscillatory van der Pol system. Appl. Math. Comput. 220, 659-667 (2013)

27. Yi, M., Huang, J.: Wavelet operational matrix method for solving fractional differential equations with variable coefficients. Appl. Math. Comput. 230, 383-394 (2014)

28. Saeed, U., Rejman, M., lqubal, M.: Haar wavelet-Picard technique for fractional order nonlinear initial and boundary value problems. Sci. Res. Essays 9(12), 571-580 (2014)

29. Saeed, U., ur Rehman, M.: Haar wavelet Picard method for fractional nonlinear partial differential equations. Appl. Math. Comput. 264, 310-322 (2015)

30. Majak, J., Pohlak, M., Karjust, K., Eerme, M., Kurnitski, J., Shvartsman, B.: New higher order Haar wavelet method: application to FGM structures. Compos. Struct. 201, 72-78 (2018)

31. Alderremy, A., Saad, K.M., Agarwal, P., Aly, S., Jain, S.: Certain new models of the multi space-fractional Gardner equation. Phys. A, Stat. Mech. Appl. 545, 123806 (2020)

32. Zhang, X., Agarwal, P., Liu, Z., Peng, H.: The general solution for impulsive differential equations with Riemann-Liouville fractional-order $q \in(1,2)$. Open Math. 13(1), 908-923 (2015)

33. Agarwal, P., Singh, R.: Modelling of transmission dynamics of Nipah virus (NIV): a fractional order approach. Phys. A, Stat. Mech. Appl. 547, 124243 (2020)

34. Morales-Delgado, V., Gómez-Aguilar, J., Saad, K.M., Khan, M.A., Agarwal, P.: Analytic solution for oxygen diffusion from capillary to tissues involving external force effects: a fractional calculus approach. Phys. A, Stat. Mech. Appl. 523, 48-65 (2019)

35. Choi, J., Agarwal, P.: A note on fractional integral operator associated with multiindex Mittag-Leffler functions. Filomat 30(7), 1931-1939 (2016)

36. Agarwal, P., Jleli, M., Samet, B.: Fixed Point Theory in Metric Spaces. Recent Advances and Applications (2018)

37. Agarwal, P., Jain, S., Mansour, T.: Further extended Caputo fractional derivative operator and its applications. Russ. J. Math. Phys. 24(4), 415-425 (2017)

38. Amiri, S., Hajipour, M., Baleanu, D.: On accurate solution of the Fredholm integral equations of the second kind. Appl. Numer. Math. 150, 478-490 (2020)

39. Soltanpour Moghadam, A., Arabameri, M., Baleanu, D., Barfeie, M.: Numerical solution of variable fractional order advection-dispersion equation using Bernoulli wavelet method and new operational matrix of fractional order derivative. Math. Methods Appl. Sci. 43(7), 3936-3953 (2020)

40. Farooq, U., Khan, H., Baleanu, D., Arif, M.: Numerical solutions of fractional delay differential equations using Chebysher wavelet method. Comput. Appl. Math. 38(4), 195 (2019)

41. Khalil, H., Khan, R.A., Baleanu, D., Rashidi, M.M.: Some new operational matrices and its application to fractional order Poisson equations with integral type boundary constrains. Comput. Math. Appl. 78(6), 1826-1837 (2019)

42. Arbabi, S., Nazari, A., Darvishi, M.T.: A two-dimensional Haar wavelets method for solving systems of PDEs. Appl. Math. Comput. 292, 33-46 (2017)

43. Haq, S., Ghafoor, A.: An efficient numerical algorithm for multi-dimensional time dependent partial differential equations. Comput. Math. Appl. 75(8), 2723-2734 (2018)

\section{Submit your manuscript to a SpringerOpen ${ }^{\circ}$ journal and benefit from:}

- Convenient online submission

- Rigorous peer review

- Open access: articles freely available online

- High visibility within the field

- Retaining the copyright to your article

Submit your next manuscript at $\gg$ springeropen.com 